\title{
Herschel and IRAM-30 m observations of comet C/2012 S1 (ISON) at 4.5 AU from the Sun ${ }^{\star}$
}

\author{
L. O’Rourke ${ }^{1}$, D. Bockelée-Morvan ${ }^{2}$, N. Biver ${ }^{2}$, B. Altieri ${ }^{1}$, D. Teyssier ${ }^{1}$, L. Jorda ${ }^{3}$, V. Debout ${ }^{2}$, C. Snodgrass ${ }^{4}$, \\ M. Küppers ${ }^{1}$, M. A’Hearn ${ }^{5}$, T. G. Müller ${ }^{6}$, and T. Farnham ${ }^{5}$

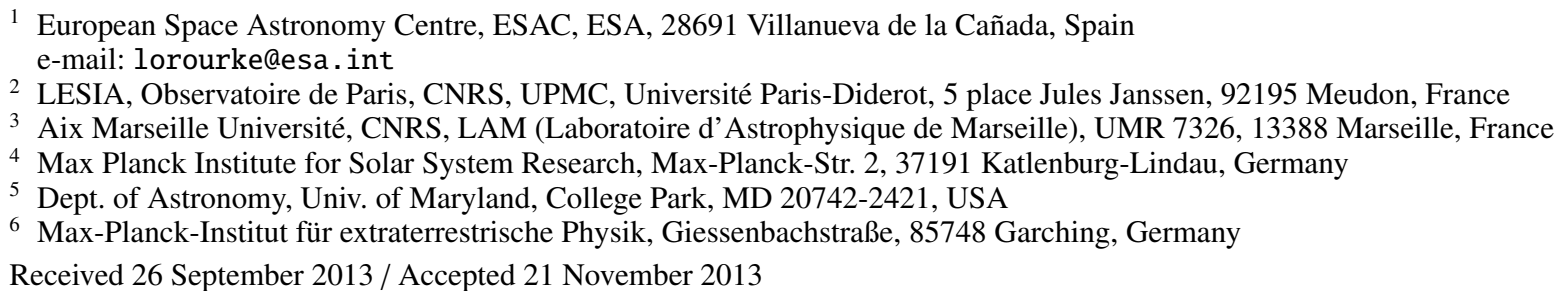

\section{ABSTRACT}

Context. The sungrazer comet C/2012 S1 (ISON) (perihelion at $r_{\mathrm{h}}=0.0125 \mathrm{AU}$ from the Sun) was bright and active when discovered in September 2012 at 6.3 AU from the Sun.

Aims. Our goal was to characterize the distant gaseous and dust activity of this comet, inbound, from observations of $\mathrm{H}_{2} \mathrm{O}, \mathrm{CO}$ and the dust coma in the far-infrared and submillimeter domains.

Methods. We report observations undertaken with the Herschel space observatory on 8 and 13 March $2013\left(r_{\mathrm{h}}=4.54-4.47 \mathrm{AU}\right)$ and with the $30 \mathrm{~m}$ telescope of Institut de Radioastronomie Millimétrique (IRAM) in March and April $2013\left(r_{\mathrm{h}}=4.45-4.18\right.$ AU). The HIFI instrument aboard Herschel was used to observe the $\mathrm{H}_{2} \mathrm{O} 1_{10}-1_{01}$ line at $557 \mathrm{GHz}$, whereas images of the dust coma at $70 \mu \mathrm{m}$ and $160 \mu \mathrm{m}$ were acquired with the PACS instrument. Spectra acquired at the IRAM $30 \mathrm{~m}$ telescope cover the CO J(2-1) line at $230.5 \mathrm{GHz}$. The spectral observations were analysed with excitation and radiative transfer models. A model of dust thermal emission taking into account a range of dust sizes is used to analyse the PACS maps.

Results. While $\mathrm{H}_{2} \mathrm{O}$ was not detected in our 8 March 2013 observation, we derive a sensitive $3 \sigma$ upper limit of $Q_{\mathrm{H}_{2} \mathrm{O}}<3.5 \times$ $10^{26}$ molecules s ${ }^{-1}$ for this date. A marginal $3.2 \sigma$ detection of $\mathrm{CO}$ is found, corresponding to a CO production rate of $Q_{\mathrm{CO}}=3.5 \times$ $10^{27}$ molecules s$^{-1}$. The Herschel PACS measurements show a clear detection of the coma and tail in both the $70 \mu \mathrm{m}$ and $160 \mu \mathrm{m}$ maps. Under the assumption of a 2-km radius nucleus, we infer dust production rates in the range $10-13 \mathrm{~kg} \mathrm{~s}^{-1}$ or $40-70 \mathrm{~kg} \mathrm{~s}^{-1}$, depending on whether a low or high gaseous activity from the nucleus surface is assumed. We constrain the size distribution of the emitted dust by comparing PACS 70 and $160 \mu \mathrm{m}$ data, and considering optical data. Size indices between -4 and -3.6 are suggested. The morphology of the tail observed on $70 \mu \mathrm{m}$ images can be explained by the presence of grains with ages older than 60 days.

Key words. comets: general - Oort Cloud - comets: individual: C/2012 S1 (ISON)

\section{Introduction}

Comet C/2012 S1 (ISON) was discovered in September 2012 at $r_{\mathrm{h}}=6.3 \mathrm{AU}$ from the Sun by the Russian astronomers Vitali Nevski and Artyom Novichonok (Nevski \& Novichonok 2012) using a 15.7-inch reflecting telescope of the International Scientific Optical Network (ISON) near Kislovodsk. Its orbit is nearly parabolic, consistent with a dynamically new comet coming freshly from the Oort cloud. It is peculiar in that it is a sungrazer $(q=0.012 \mathrm{AU}$ on 28 November 2013) although not of the Kreutz group.

The comet passed approximately 0.072 AU from Mars on 1 October 2013 and passes approximately 0.42 AU from Earth on 26 December 2013. Because of its distant activity and expected exceptional brightness at perihelion, comet C/2012 S1 (ISON) is the object of a worldwide campaign involving many space and ground-based observatories.

* Herschel is an ESA space observatory with science instruments provided by European-led Principal Investigator consortia and with important participation from NASA. Based on observations carried out with the $30 \mathrm{~m}$ telescope, operated by the Institut de RadioAstronomie Millimétrique (IRAM), which is funded by a partnership of INSU/CNRS (France), MPG (Germany), and IGN (Spain).
This paper covers observations of comet C/2012 S1 (ISON) undertaken in March and April $2013\left(r_{\mathrm{h}}=4.5-4.2 \mathrm{AU}\right)$ in the far-infrared with the Herschel Space Observatory (Pilbratt et al. 2010) using director discretionary time and observations from ground at millimetric wavelengths obtained with the $30 \mathrm{~m}$ antenna of the Institut de RadioAstronomie Millimétrique (IRAM).

We first present in Sect. 2 the observations performed with the Heterodyne Instrument for the Far Infrared (HIFI, de Graauw et al. 2010) of comet C/2012 S1 (ISON) and the data analysis performed on that data set. An upper limit on the water production rate is derived. This is followed in Sect. 3 by an analysis of the IRAM search for $\mathrm{CO}$ whereby a marginal detection is described. Section 4 presents images of the dust coma obtained with the Photodetector Array Camera and Spectrometer (PACS, Poglitsch et al. 2010), which serve to derive the dust production rate and constrain its size distribution. Section 5 outlines the conclusions we derive from the work performed in the paper.

\section{Herschel HIFI observations search for $\mathrm{H}_{2} \mathrm{O}$}

Comet C/2012 S1 (ISON) was observed with the HIFI instrument on Herschel on 8 March 2013 UT (ObsId: 1342266412) with a total on-target integration time of $2.9 \mathrm{~h}$ and a line of sight 
Table 1. C/2012 S1 (ISON) $\mathrm{H}_{2} \mathrm{O}$ and $\mathrm{CO}$ observations.

\begin{tabular}{lcclccccccc}
\hline \hline Line & $\begin{array}{c}v \\
(\mathrm{GHz})\end{array}$ & $\begin{array}{l}\text { Date }(\mathrm{UT}) \\
\text { yyyy/mm/dd.dd }\end{array}$ & $\begin{array}{c}r_{\mathrm{h}}{ }^{a} \\
(\mathrm{AU})\end{array}$ & $\begin{array}{c}\Delta^{b} \\
(\mathrm{AU})\end{array}$ & $\begin{array}{c}\phi^{c} \\
\left.{ }^{\circ}\right)\end{array}$ & $\begin{array}{c}\text { Integ. } \\
\text { time }(\mathrm{min})\end{array}$ & $\begin{array}{c}\text { Line area } \\
\left(\mathrm{mK} \mathrm{km} \mathrm{s}^{-1}\right)\end{array}$ & \multicolumn{2}{c}{$\begin{array}{c}\text { Production rate }\left(\mathrm{mol} . \mathrm{s}^{-1}\right) \\
T_{\text {kin }}=8 \mathrm{~K}\end{array}$} & $T_{\text {kin }}=20 \mathrm{~K}$ \\
\hline $\mathrm{H}_{2} \mathrm{O}$ & $1_{10}-1_{01}$ & 556.936 & $2013 / 03 / 08.13$ & 4.54 & 4.07 & 11.8 & 177 & $<5.4^{e}$ & $<4.8 \times 10^{26 f}$ \\
$\mathrm{CO}$ & $2-1$ & 230.538 & $2013 / 03 / 15.70$ & 4.45 & 4.10 & 12.5 & 21 & $40 \pm 18$ & $4.5 \pm 2.0 \times 10^{27}$ & $3.8 \pm 1.7 \times 10^{26}$ \\
$\mathrm{CO}$ & $2-1$ & 230.538 & $2013 / 04 / 06.66-08.67$ & 4.18 & 4.22 & 13.7 & 47 & $32 \pm 13$ & $3.5 \pm 1.4 \times 10^{27}$ & $2.9 \pm 1.2 \times 10^{27}$ \\
$\mathrm{CO}$ & $2-1$ & 230.538 & $2013 / 03 / 15.7-04 / 08.7$ & 4.27 & 4.19 & 13.0 & 68 & $38 \pm 12$ & $4.2 \pm 1.3 \times 10^{27}$ & $3.5 \pm 1.1 \times 10^{27}$ \\
\hline
\end{tabular}

Notes. ${ }^{(a)} r_{\mathrm{h}}$ is the comet's heliocentric distance at the time of observation. ${ }^{(b)} \Delta$ is the distance from target to the observer. ${ }^{(c)}$ Phase Angle. ${ }^{\left({ }^{(d)}\right.}$ Line area in the main beam brightness temperature scale computed in a $(-0.6,+0.6) \mathrm{km} \mathrm{s}^{-1}$ window. ${ }^{(e)}$ From the HRS spectrum, the upper limit from the WBS spectrum is $4.2 \mathrm{mK} \mathrm{km} \mathrm{s}^{-1}$. ${ }^{(f)}$ The values provided for the gas production rates are $3 \sigma$ upper limits.

velocity (delta-dot) of $8.24 \mathrm{~km} \mathrm{~s}^{-1}$. Further geometric circumstances are presented in Table 1.

We searched in the upper sideband of the HIFI band 1a mixer for line emission from the fundamental ortho- $\mathrm{H}_{2} \mathrm{O} 1_{10}-1_{01}$ line at $556.936 \mathrm{GHz}$. Our observation was performed using both the Wide Band Spectrometer (WBS) and the High Resolution Spectrometer (HRS). The spectral resolution of the WBS is $1 \mathrm{MHz}\left(0.54 \mathrm{~km} \mathrm{~s}^{-1}\right.$ at the frequency of the observed line), while the HRS was used in its high-resolution mode with a resolution of $120 \mathrm{kHz}\left(0.065 \mathrm{~km} \mathrm{~s}^{-1}\right)$. The observing mode used was the frequency-switching observing mode with a frequency throw of 94.5 $\mathrm{MHz}$ and without a reference position on the sky. While the advantage of using this mode is that the on-target integration time is maximized, the downside is that the statistical noise can be underestimated due to strong baseline ripples and uncertainties in their removal.

Initial processing of the HIFI data set was performed using the standard HIFI processing pipeline of HIPE v9.2 (Herschel interactive processing environment, Ott 2010). This processing served to reduce the data to calibrated level- 2 data products. The main beam brightness temperature scale was computed using a forward efficiency of 0.96 and beam efficiency of 0.75 . Vertical and horizontal polarizations were averaged weighted by the mean square amplitude in order to increase the signal-tonoise ratio. The pointing offset of both the vertical and the horizontal polarization spectra is $3.3^{\prime \prime}$ in band 1a when compared to the target position. The Half Power Beam Width (HPBW) is $38.1^{\prime \prime}$ at $557 \mathrm{GHz}$. No hint of an $\mathrm{H}_{2} \mathrm{O}$ line is found in the resultant spectra. We show the HRS averaged spectrum of the two orthogonal polarisations in Fig. 1.

To derive an upper limit on the water production rate, we used a molecular excitation model which calculates the population of the rotational levels of water as a function of nucleocentric distance. The excitation model includes collisions of water and electrons in the inner coma, infrared pumping of the vibrational bands, and treats self-absorption using the escape probability formalism (e.g., Biver et al. 2007; Zakharov et al. 2007). Model calculations were performed with input parameters which include the electron density, the gas expansion velocity, and the gas kinetic temperature $T_{\text {kin }}$ (used to control the molecular excitation in the collisional region). Synthetic spectra were computed considering the transfer of line radiation of ortho-water in the cometary atmosphere. Since the electron density in the coma is not well constrained, an electron density scaling factor of $x_{n_{\mathrm{e}}}=0.2$ with respect to the standard profile derived from observations of comet 1P/Halley has been used (e.g., Hartogh et al. 2010). For the radial gas density profile, we adopted the standard spherically symmetric Haser distribution. We assumed an expansion velocity of $0.35 \mathrm{~km} \mathrm{~s}^{-1}$ derived from the CO $J(2-1)$ line profile (Sect. 3) following similar techniques as described in, e.g.

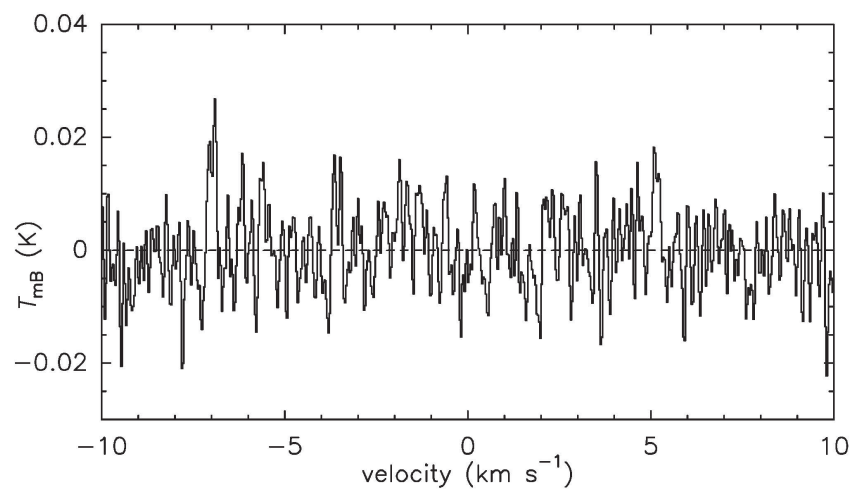

Fig. 1. Averaged spectrum of the two orthogonal polarisations of the $\mathrm{H}_{2} \mathrm{O}$ line $1_{10}-1_{01}$ at $556.936 \mathrm{GHz}$ towards comet $\mathrm{C} / 2012 \mathrm{~S} 1$ (ISON) obtained by Herschel/HIFI on 8.1 March 2013 UT with the HRS spectrometer. The vertical scale is the main-beam brightness temperature. The horizontal scale is the Doppler velocity in the comet rest frame.

Biver et al. (2002) and O'Rourke al. (2013). The upper limit on the water production rate was calculated against two different values of the kinetic temperature, $8 \mathrm{~K}$ and $20 \mathrm{~K}$. The $8 \mathrm{~K} \mathrm{ki-}$ netic temperature value is inferred from that derived for comet 29P/Schwassmann-Wachmann 1 (Gunnarsson et al. 2008) and the $20 \mathrm{~K}$ temperature value from that derived for comet HaleBopp (Biver et al. 2002) when both were at the same heliocentric distance as ISON at the time of the observations.

Table 1 provides the $3 \sigma$ upper limit on the line integrated intensity and the corresponding production rate. For $T_{\text {kin }}=$ $20 \mathrm{~K}$, we derive a sensitive $3 \sigma$ upper limit of $Q_{\mathrm{H}_{2} \mathrm{O}}<3.5 \times$ $10^{26}$ molecules s ${ }^{-1}$.

\section{IRAM observations of $\mathrm{CO}$}

Comet C/2012 S1 (ISON) was observed with the IRAM $30 \mathrm{~m}$ radio telescope on 15 March, 6 and 8 April 2013 UT (see Table 1 for the geometric circumstances of the observations). These were short observations inserted in the pre-existing schedule. Observations were done using the wobbling secondary mirror in March, and in position switching mode in April with a reference sky at $3^{\prime}$ from the comet. The EMIR $230 \mathrm{GHz}$ receiver was used, and spectra covering $224.9-232.6 \mathrm{GHz}$ and 240.6-248.3 GHz were obtained with the FTS spectrometer at a resolution of $200 \mathrm{kHz}$. In addition, the Vespa autocorrelator covered the $\mathrm{CO} J(2-1)$ line at $230.538 \mathrm{GHz}$ with a resolution of $40 \mathrm{kHz}\left(52 \mathrm{~m} \mathrm{~s}^{-1}\right.$, Fig. 2). The IRAM beam size is $10.7^{\prime \prime}$ (HPBW) at $230 \mathrm{GHz}$.

A marginal narrow signal was seen at the comet velocity in both periods and with both spectrometers, but the detection level 
Table 2. PACS observations of comet C/2012 S1 (ISON).

\begin{tabular}{lccccccc}
\hline \hline $\begin{array}{l}\text { Wavelength } \\
(\mu \mathrm{m})\end{array}$ & $\begin{array}{c}\text { Date }(\mathrm{UT}) \\
(\mathrm{yyyy} / \mathrm{mm} / \mathrm{dd} . \mathrm{dd})\end{array}$ & $\begin{array}{c}r_{\mathrm{h}} \\
(\mathrm{AU})\end{array}$ & $\begin{array}{c}\Delta \\
(\mathrm{AU})\end{array}$ & $\begin{array}{c}\phi \\
\left({ }^{\circ}\right)\end{array}$ & $\begin{array}{c}\text { Integ. } \\
\text { time }(\mathrm{min})\end{array}$ & $\begin{array}{c}\text { Total flux }^{a} \\
(\mathrm{mJy})\end{array}$ & $\begin{array}{c}\text { Flux at photocentre }^{a, b} \\
(\mathrm{mJy} / \mathrm{pxl})\end{array}$ \\
\hline 70 & $2013 / 03 / 13.8$ & 4.47 & 4.10 & 12.5 & 56.5 & $188 \pm 9$ & $1.48 \pm 0.07$ \\
160 & $2013 / 03 / 13.8$ & 4.47 & 4.10 & 12.5 & 56.5 & $51 \pm 3$ & $0.72 \pm 0.04$ \\
\hline
\end{tabular}

Notes. ${ }^{(a)}$ Colour-corrected flux in an aperture of $25^{\prime \prime}$ radius, with the sky reference between 40 and $50^{\prime \prime}$. The quoted uncertainty is $5 \%$ corresponding to the uncertainty in the flux calibration. ${ }^{(b)}$ The pixel size is 1 and $2^{\prime \prime}$, at 70 and $160 \mu \mathrm{m}$, respectively.

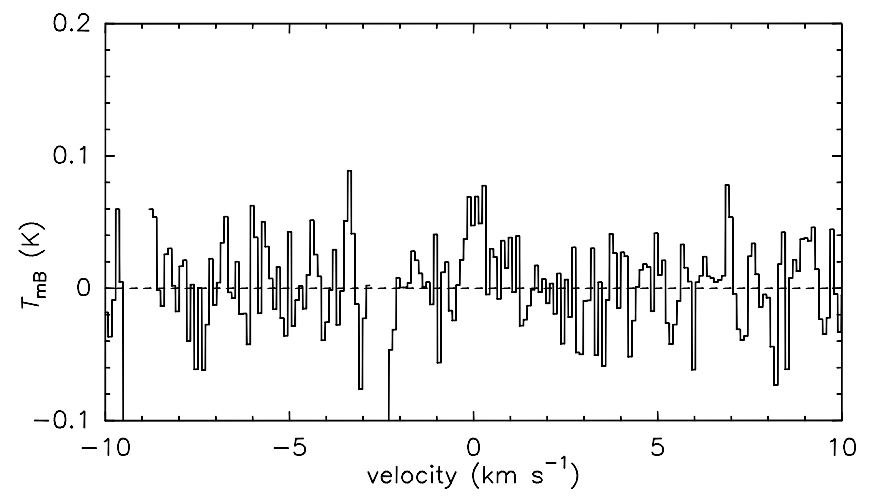

Fig. 2. Average CO $J(2-1)$ spectrum obtained with the IRAM $30 \mathrm{~m}$ radio telescope between 15.7 March and 8.7 April 2013, using the high resolution Vespa autocorrelator. The vertical scale is the main-beam brightness temperature. The horizontal scale is the Doppler velocity in the comet rest frame.

in the average spectrum does not exceed $3-4 \sigma$ (Fig. 2). No other line (e.g., from $\mathrm{CS}, \mathrm{H}_{2} \mathrm{CO}, \mathrm{CH}_{3} \mathrm{OH}$ ) was seen.

The line width suggests a rather low expansion velocity $\left(0.35 \mathrm{~km} \mathrm{~s}^{-1}\right)$ Biver et al. (2002), which is used to derive the $\mathrm{CO}$ production rate. The line integrated intensity and corresponding production rate for $T_{\text {kin }}=8$ and $20 \mathrm{~K}$ are given in Table 1 . For $T_{\text {kin }}=20 \mathrm{~K}$, we derive a CO production rate $Q_{\mathrm{CO}}=$ $3.5 \times 10^{27}$ molecules s $^{-1}$. The value derived for $T_{\text {kin }}=8 \mathrm{~K}$ is of the same order.

\section{Herschel PACS data}

\subsection{Observations}

Comet C/2012 S1 (ISON) was observed by the Herschel PACS instrument on 13 March 2013 UT with a total on-target integration time of $1.9 \mathrm{~h}$. The geometric circumstances are presented in Table 2.

The observations were performed in the $70-\mu \mathrm{m} / 160-\mu \mathrm{m}$ filter combination (so-called blue/red bands). Two observation pairs (ObsId: 1342267433-34; 1342267443-44) were performed ( 2 scan directions, $70^{\circ}$ and $110^{\circ}$ ) separated by approximately $1.5 \mathrm{~h}$ to ensure that the comet had moved during the course of the full observation, supporting easier extraction of the comet signal from the background. Such a strategy was applied in support of data processing of Herschel observations of transNeptunian objects (TNOs; e.g., Kiss et al. 2013). We selected six repetitions in each of the two scan-directions for a better characterisation of the background. The PACS measurements were processed using HIPE v9.2 (Ott 2010). Further processing was then performed to merge the four ObsIds for each of the two filters and correct for the motion of the comet during the course of the observation. We used a highpass filter of 50 frames/readout at both wavelengths to remove $1 / f$ noise. Figure 3 provides the two resulting blue and red contour maps.

Using the "Annular Sky Aperture" task of HIPE, the fluxes within a $25^{\prime \prime}$-radius aperture centred on maximum brightness were extracted for both maps, with the background being subtracted via the same task. The derived fluxes were colour corrected (1.0 at $70 \mu \mathrm{m}$ and 1.06 at $160 \mu \mathrm{m}$; Müller et al. 2011) to obtain monochromatic flux densities at the PACS reference wavelengths. Table 2 provides the Herschel PACS observation dataset and accompanying observation information including the final derived total fluxes. We also provide the flux density measured at the photocentre of the images.

\subsection{Dust coma and tail morphology}

In both the blue and red maps, there is clear extended emission - although less so in the red $160-\mu \mathrm{m}$ map. Original assumptions that the red map might provide an estimate on the object size were discarded based upon our use of a Thermophysical Model (O'Rourke al. 2012; Müller et al. 2012). Indeed, the radius of comet ISON's nucleus has been measured to be less than $R_{\mathrm{N}}=2 \mathrm{~km}$ from measurements with the Hubble Space Telescope ( $\mathrm{Li}$ et al. 2013). Assuming a low albedo (0.03) inert object with a maximum radius of $2 \mathrm{~km}$, with the same observing conditions (distance, phase angle) as PACS, the expected nucleus flux is about $1.5-2 \mathrm{mJy}$ at $70 \mu \mathrm{m}$, i.e., well below the observed signal (Table 2). At $160 \mu \mathrm{m}$ the object emission would be below $0.5 \mathrm{mJy}$ approximatively. Effectively, in both cases, the nucleus signal falls within the noise, and the detected signal is from emitted dust. In that respect, we exclude any detection of the nucleus in our observations and focus on the flux measured being primarily from the dust coma and the tail.

At the time of the PACS observations, the position angle of the extended Sun-target radius vector was $96.6^{\circ}$, and the negative of the heliocentric velocity vector, as seen in the observer's plane-of-sky, was $83.2^{\circ}$. The tail direction in the blue and red images is along the Sun-comet vector (Fig. 3), as observed on optical images (Meech et al. 2013). The coma is clearly less extended in the $160 \mu \mathrm{m}$ image (Fig. 3).

We studied the mean radial profile of inner dust-coma emission, performing azimuthal averages. The width of the radial profile in the 70- $\mu \mathrm{m}$ map is $9.6^{\prime \prime}$, consistent with the expected width for an $1 / \rho$ brightness distribution convolved with the point spread function (PSF) (half width at half maximum of 5.5", Lutz 2012), where $\rho$ is the distance to the nucleus. For the $160-\mu \mathrm{m}$ map, the width is $\sim 12^{\prime \prime}$, i.e., on the order of the PSF at this wavelength $\left(11^{\prime \prime}\right)$, whereas a width of $17^{\prime \prime}$ is expected for steady state dust production, not considering dynamics effects (Bockelée-Morvan et al. 2010). This suggests an excess of slow large particles in the $160-\mu \mathrm{m}$ image. With the expected terminal velocity of $100-\mu \mathrm{m}$ dust particles being typically $10 \mathrm{~m} \mathrm{~s}^{-1}$ using our dust models described below, we estimate that the travel time of these particles through the PSF is $\sim 15$ days. 

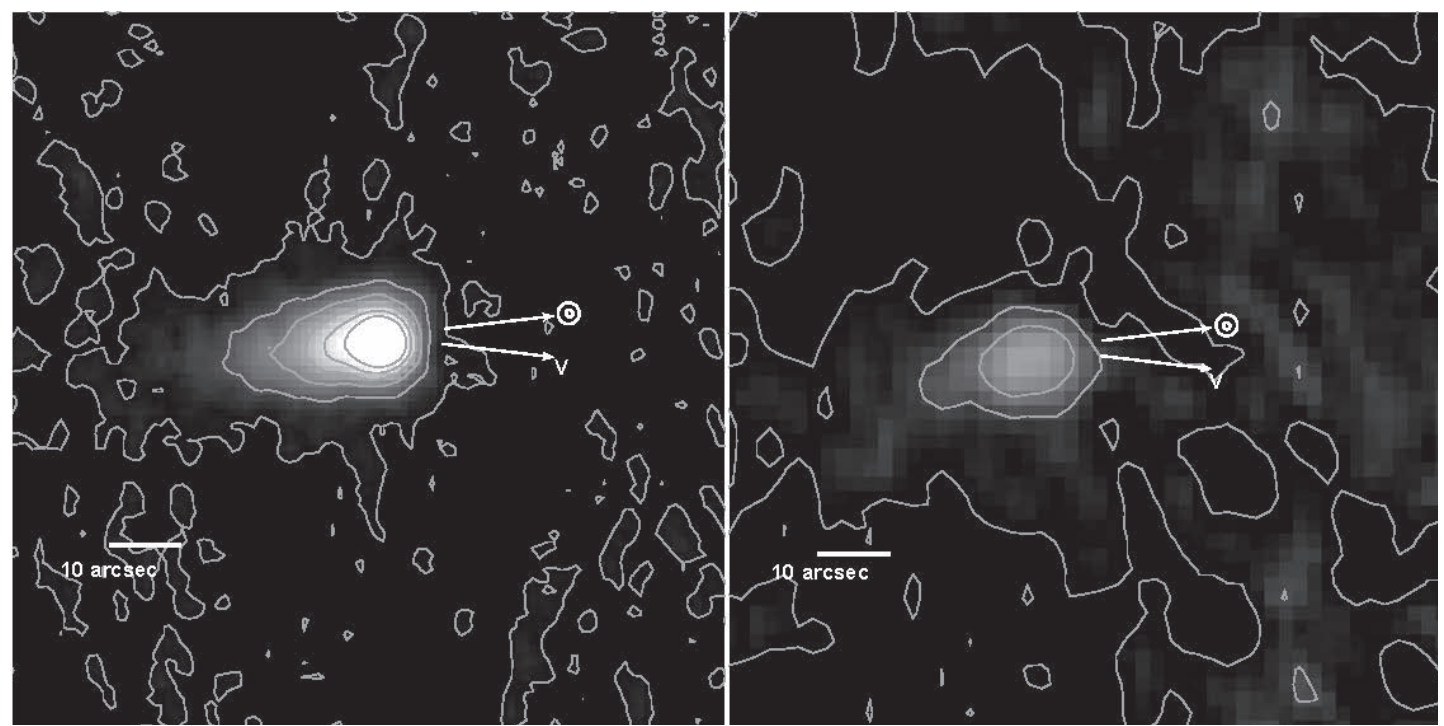

Fig. 3. $70 \mu \mathrm{m}$ (left, blue map) and $160 \mu \mathrm{m}$ (right, red map) images of comet C/2012 S1 (ISON) observed with PACS on 13 March 2013 UT. The pixel size is $1^{\prime \prime}$ for the blue map and $2^{\prime \prime}$ for the red map. Contour levels are stepped by 0.002 in Log, up to $99 \%$ of maximum intensity. North is up and east to the left. The anti Sun direction is at $\mathrm{PA}=96.6^{\circ}$. The arrows indicate the sun direction and projected comet velocity vector. The size of the blue and red images is $\sim 100^{\prime \prime}$.
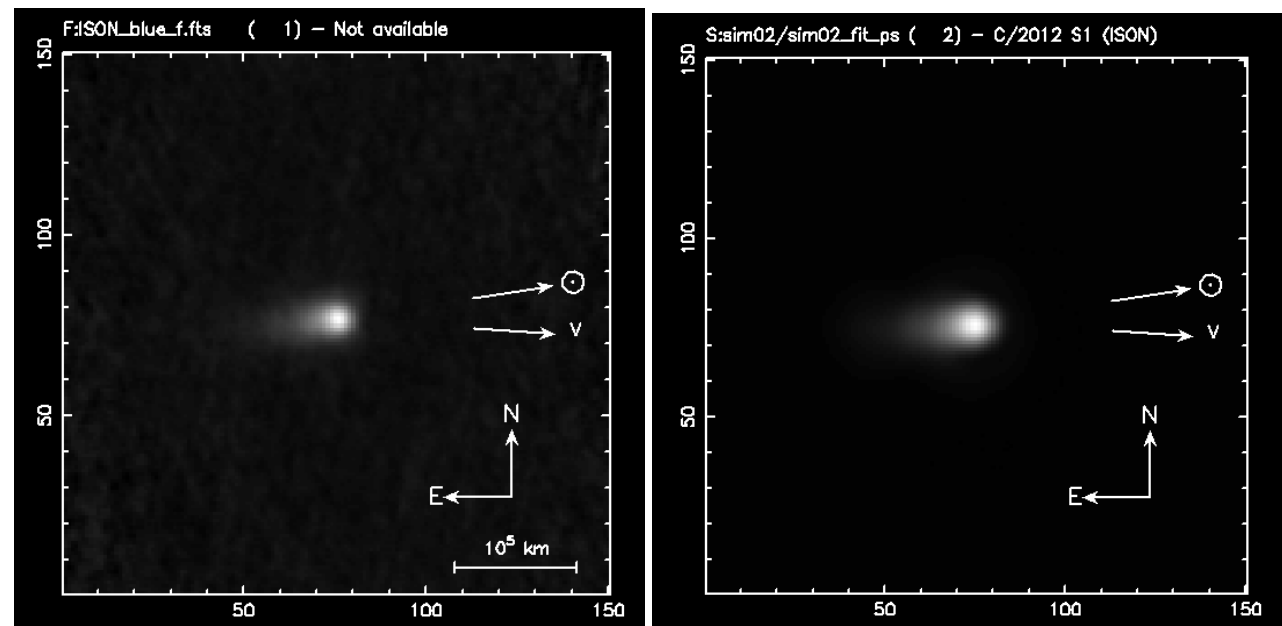

Fig. 4. A comparison of the dust tail of comet C/2012 S1 (ISON) observed in the blue channel of PACS (left image) with a dynamical model of the dust tail (right image). For details of the model, see text. The direction of the Sun and that of the projected velocity vector of the comet are indicated with arrows. The axes units are in arcseconds.

We performed a dynamical simulation of the coma of comet C/2012 S1 (ISON) to explain the structure of the dust tail. We used a Monte-Carlo program which computes the trajectory of dust grains under reduced gravity, following a FinsonProbstein approach. The relationship between the $\beta$ coefficient and the radius of the grains has been calculated assuming spherical olivine dust particles $\left(\mathrm{MgFeSiO}_{4}\right)$ and Mie theory, where $\beta$ is equal to the ratio of the accelerations due to radiation pressure and solar gravity (Burns et al. 1979). We assume hereafter that particles with a diameter $2 \pi a<\lambda$ do not contribute significantly to the infrared signal because their absorption efficiency factor $Q_{\text {abs }}(a, \lambda)$ drops rapidly below this limit (van de Hulst 1981). We therefore consider a distribution of dust particles with radii $a=11-100 \mu \mathrm{m}$, velocities $v_{\mathrm{d}}=0.04-4 \mathrm{~m} \mathrm{~s}^{-1}$ and age $\tau_{\mathrm{d}}<360$ days. The size of the test particles corresponds to $\beta=0.0015-0.015$. The maximum terminal dust velocity of $4 \mathrm{~m} \mathrm{~s}^{-1}$ has been calculated using a "trial and test" process. We used synthetic images of the dust tail for a wide range of size and velocity, which we compared visually to the PACS image to determine what dust velocities could reproduce both the extension of the dust coma in the solar direction and the width of the dust tail in the anti-solar direction. The minimum terminal velocity has been chosen arbitrarily, and we could not constrain its value from our simulations. The output of the simulation is a series of 1000 images, in units of number of grains, for 10 bins of sizes, dust velocities and delays (see Jorda et al. 2007). The images are combined linearly to reproduce the spatial distribution of dust observed in the blue PACS image. The results of the comparison are illustrated in Fig. 4 (right panel), which shows our model of the dust tail.

The tail is observed to have a projected length of up to $70000 \mathrm{~km}$ in the anti-solar direction (see Fig. 4, left panel). Our simulation shows that grains with ages $\tau \geq 60$ days are required to reproduce the observed length of the dust tail. This is explained by the weak radiation pressure at such large distances from the Sun combined with the low values of the $\beta$ parameter. In our simulation, the unresolved central pixels contain grains of ages equal to 2-20 days, depending on their terminal velocities 
consistent with our analysis of the red image above. The grains described above would be the large dust distribution counterpart of the smaller micron-size dust observed in the optical tail by Meech et al. (2013) between February and May 2013.

\subsection{Dust production rates and size distribution from PACS data}

Analysis of the ISON PACS images to determine the dust production rate $Q_{\text {dust }}$ was performed by comparing the flux density measured on the brightest pixels of the blue and red maps (Table 2) with that of a model of dust thermal emission.

The model used for this study is the same as that applied to an equivalent PACS data set of comet C/2006 W3 (Christensen) (Bockelée-Morvan et al. 2010), and of the Centaurs (2060) Chiron and (10199) Chariklo (Fornasier et al. 2013). The basic principles of this model are given in Jewitt \& Luu (1990). Absorption cross-sections calculated with the Mie theory are used to compute both the temperature of the grains, solving the equation of radiative equilibrium, and their thermal emission. Complex refractive indices of amorphous carbon and olivine $(\mathrm{Mg}: \mathrm{Fe}=50: 50)($ Edoh 1983; Dorschner et al. 1995) are taken as broadly representative of cometary dust.

We consider a differential dust production $Q_{\text {dust }}(a)$ as a function of grain radius $a$, described by the size index $\alpha$. The sizedependent grain velocities $v_{\mathrm{d}}(a)$, as well as the maximum grain radius $a_{\mathrm{max}}$, are computed following Crifo \& Rodionov (1997). We assume a nucleus and dust density of $500 \mathrm{~kg} \mathrm{~m}^{-3}$ (Thomas et al. 2013; Davidsson et al. 2007; Richardson et al. 2007; Niimi et al. 2012). Both the maximum grain size and dust velocities critically depend on the nucleus size and the gas production rate at the surface, which are both poorly constrained. For the nucleus size, as a nominal value, our model assumes a nucleus radius of $R_{\mathrm{N}}=2 \mathrm{~km}$, consistent with the size limit inferred by Li et al. (2013). For the gas production rate, we utilise as input our results from the HIFI and IRAM measurements.

Recognising that the HIFI inferred water production rate is at least one order of magnitude weaker than the $\mathrm{CO}$ production rate (Table 1), we assume a CO-dominated coma with a $\mathrm{CO}$ production rate of $4 \times 10^{27}$ molecules $\mathrm{s}^{-1}$, corresponding to the value measured at IRAM on 15 March 2013 (Table 1). However, we would normally expect the $\mathrm{CO}_{2}$ production rate to compete with or even dominate the $\mathrm{CO}$ production, based on measurements of the $\mathrm{CO}$ and $\mathrm{CO}_{2}$ production rates in other comets at large heliocentric distances (Crovisier et al. 1997; Biver et al. 2002; Ootsubo et al. 2012). With no measurement of the $\mathrm{CO}_{2}$ production rate existing for comet ISON in the March 2013 timeframe, and the IRAM marginal detection not confirmed by observations undertaken at the James Clerk Maxwell Telescope (Meech et al. 2013), we look to Spitzer observations from the June 2013 timeframe to contribute to our analysis.

Lisse et al. (2013) suggest a $\mathrm{CO}_{2}$ production rate of $1.9 \times 10^{26}$ molecules s $^{-1}$ for 13 June $\left(r_{\mathrm{h}}=3.34 \mathrm{AU}\right)$, well below the CO production rate measured on 15 March 2013. Meech et al. (2013) suggested that the activity of comet ISON was $\mathrm{CO}_{2}$ dominated at large heliocentric distances inbound but experienced a long slow outburst between 6-3.5 AU triggered by the sublimation of CO from deep layers. One cannot rule out however that the high $\mathrm{CO}$ production rate we measured in March 2013, if real, might be related to sublimation from icy grains exposing a large cross-section to Sun radiation.

For completeness therefore, and based upon the above analysis, we have also considered a gas production rate from the surface ten times lower than measured at IRAM i.e., equal to
$4 \times 10^{26}$ molecules s $^{-1}$. For comparison, this value is slightly larger than the value of $2.5 \times 10^{26}$ molecules $\mathrm{s}^{-1}$ deduced from the correlation between the heliocentric magnitude and the $\mathrm{CO}$ production rate observed in comet Hale-Bopp (Biver 2001). Note that we assume a CO-dominated activity, but similar results are obtained for the dust production rate using $\mathrm{CO}_{2}$ instead.

Meech et al. (2013) conducted Finson-Probstein modelling (Finson \& Probstein. 1968) of the dust tail of comet ISON, and deduced a dust expansion speed of $10 \mathrm{~m} \mathrm{~s}^{-1}$ assuming that micron-sized dust grains dominated the optical appearance of the comet. Such a low velocity would be more consistent with a low surface activity (our dust model predicts a velocity of $35 \mathrm{~m} \mathrm{~s}^{-1}$ for $1-\mu \mathrm{m}$ radius particles in the low activity case, Table 3 ). Similarly, our Finson-Probstein modelling of the PACS $70 \mu \mathrm{m}$ image suggests low grain velocities, though these velocities pertain to old grains emitted when the comet activity was lower (Sect. 4.2).

Finally, we assumed that the dust and gas production is isotropic and that the local density of the individual dust particles follows a $1 / r^{2}$ law, where $r$ is the cometocentric distance.

Table 3 provides the dust velocity and maximum size computed by our model, and the dust production rates that match the the flux density measured on the brightest pixel of the $70 \mu \mathrm{m}$ and $160 \mu \mathrm{m}$ maps. We described the PSF by a Gaussian function of width $5.5^{\prime \prime}$ and $11^{\prime \prime}$ at $70 \mu \mathrm{m}$ and $160 \mu \mathrm{m}$, respectively (Lutz 2012). We considered a size index between -4 and -3 , which corresponds to the range of size indices measured in cometary comae.

For olivine grains, dust production rates derived from the $70 \mu \mathrm{m}$ and $160 \mu \mathrm{m}$ maps are consistent with each other within $30 \%$ in the range of size index considered, with a best fit obtained for $\alpha \sim-3.65$ to -3.6 . On the other hand, for carbon grains, the best match is obtained for a steeper size distribution with $\alpha \sim-4$. In this case there is a factor of $\sim 2$ discrepancy between $70 \mu \mathrm{m}$ and $160 \mu \mathrm{m}$ in the derived $Q_{\text {dust }}$ for $\alpha=-3$.

For $Q_{\mathrm{CO}}=4 \times 10^{27}$ molecules s ${ }^{-1}$ (i.e., $190 \mathrm{~kg} \mathrm{~s}^{-1}$ of CO) and the size index which explains both $70 \mu \mathrm{m}$ and $160 \mu \mathrm{m}$ data, we infer $Q_{\text {dust }} \sim 70 \mathrm{~kg} \mathrm{~s}^{-1}$ of olivine grains or $Q_{\text {dust }}=40 \mathrm{~kg} \mathrm{~s}^{-1}$ of carbon grains. Dust production rates are lower between 10 (model with carbon) and $13 \mathrm{~kg} \mathrm{~s}^{-1}$ (olivine), when a low gas activity at the surface of $Q_{\mathrm{CO}}=4 \times 10^{26}$ molecules s ${ }^{-1}\left(19 \mathrm{~kg} \mathrm{~s}^{-1}\right.$ of $\mathrm{CO}$ ) is considered, since the maximum grain size is then lower (Table 3).

\subsection{Combining PACS and optical data}

One can also compare the production rates derived from PACS data with the visible light proxy for dust production $A f \rho$, in units of $\mathrm{cm}$ (A'Hearn et al 1984), where $A$ is the bond albedo of the dust, $f$ is the filling factor of dust in the field-of-view, and $\rho$ is the projected radius of the field-of-view. For steady isotropic dust production expanding at constant velocity, $A f \rho$ is independent of aperture size.

Since visible and far-infrared observations of the dust coma probe particles of different sizes, combining these data may be used to constrain the index of the size distribution. Assuming single-size grains, $A f \rho$ is related to $Q_{\text {dust }}$ through (Jorda 1995):

$Q_{\text {dust }}=\frac{2}{3} A f \rho \times \frac{\rho_{\mathrm{d}} a v_{\mathrm{d}}}{A_{\mathrm{p}}}$

where $a$ is the grain radius, $\rho_{\mathrm{d}}$ is the dust density, and $A_{\mathrm{p}}$ is the geometric albedo of the dust. A more general formula can be obtained by considering a dust size distribution and a sizedependent dust velocity (Weiler et al. 2003). We used the same 
Table 3. Dust parameters and production rates for comet C/2012 S1 (ISON) derived from PACS 70 and $160 \mu \mathrm{m}$ data, and from optical data.

\begin{tabular}{|c|c|c|c|c|c|c|c|}
\hline \multirow{2}{*}{$\begin{array}{c}Q_{\mathrm{CO}} \\
\left(\text { molecules s}^{-1}\right)\end{array}$} & \multirow[t]{2}{*}{ Material } & \multirow{2}{*}{$\begin{array}{l}a_{\max } \\
(\mathrm{mm})\end{array}$} & \multirow{2}{*}{$\begin{array}{l}v_{\mathrm{d}} \\
\left(\mathrm{m} \mathrm{s}^{-1}\right)^{a}\end{array}$} & \multirow[t]{2}{*}{$\alpha$} & \multicolumn{3}{|c|}{$Q_{\text {dust }}\left(\mathrm{kg} \mathrm{s}^{-1}\right)$} \\
\hline & & & & & $70 \mu \mathrm{m}^{b}$ & $160 \mu \mathrm{m}^{b}$ & $A f \rho^{c}$ \\
\hline $4 \times 10^{27}$ & carbon/silicates & 9.2 & $1.3-167^{d}$ & -3 & $210-350^{e}$ & $150-170^{e}$ & 435 \\
\hline $4 \times 10^{27}$ & carbon/silicates & 9.2 & $1.3-167^{d}$ & -3.5 & $100-140^{e}$ & 80 & $125-176$ \\
\hline $4 \times 10^{27}$ & carbon/silicates & 9.2 & $1.3-167^{d}$ & -4 & 42 & $36-50^{f}$ & $10-35$ \\
\hline $4 \times 10^{26}$ & carbon/silicates & 0.92 & $1.3-87^{d}$ & -3 & $20-35^{e}$ & $14-16^{e}$ & 45 \\
\hline $4 \times 10^{26}$ & carbon/silicates & 0.92 & $1.3-87^{d}$ & -3.5 & $14-18^{e}$ & $11-12^{f}$ & $18-25$ \\
\hline $4 \times 10^{26}$ & carbon/silicates & 0.92 & $1.3-87^{d}$ & -4 & 12 & $10-14^{f}$ & $4-10$ \\
\hline
\end{tabular}

Notes. ${ }^{(a)}$ Dust velocities in the size range $a_{\min }-a_{\max }$, with $a_{\min }=0.1 \mu \mathrm{m} .{ }^{(b)}$ Dust production rate derived assuming $a_{\min }=0.1 \mu \mathrm{m} .{ }^{(c)}$ Dust production rate derived assuming $A f \rho=900 \mathrm{~cm}$ and $A_{\mathrm{p}}=0.04$. The values for $a_{\min }=0.1$ and $1 \mu \mathrm{m}$ are given when they differ significantly. (d) The velocity of $1-\mu \mathrm{m}$ sized particles is 35 and $85 \mathrm{~m} \mathrm{~s}^{-1}$ for $Q_{\mathrm{CO}}=4 \times 10^{26}$ and $4 \times 10^{27}$ molecules s${ }^{-1}$, respectively. ${ }^{(e)}$ The lower values correspond to olivine and higher values to carbon grains respectively. ${ }^{(f)}$ The lower values correspond to carbon and higher values to olivine grains respectively.

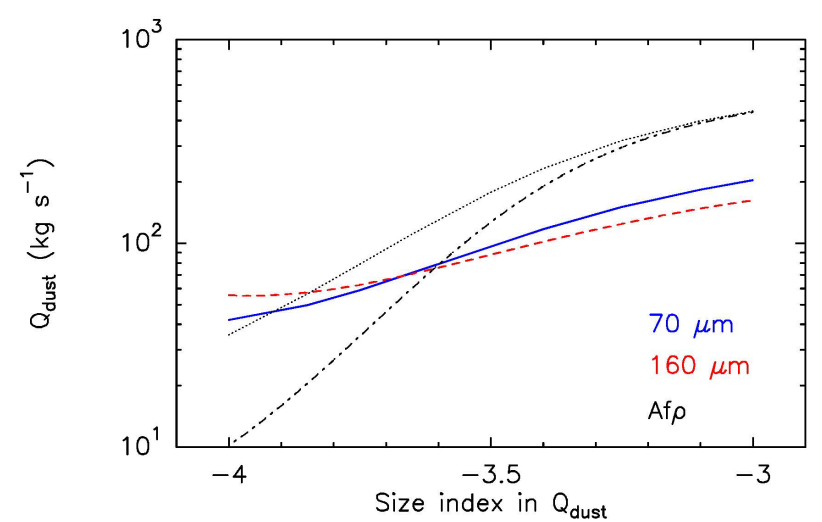

Fig. 5. Dust production rates derived from the PACS $70 \mu \mathrm{m}$ (blue curve) and $160 \mu \mathrm{m}$ (red curve) maps of comet C/2012 S1 (ISON) obtained on March 132013 UT. The black curves show values derived from $A f \rho=$ $900 \mathrm{~cm}$. Plain lines are for $a_{\min }=0.1 \mu \mathrm{m}$. Dashed lines are for $a_{\min }=$ $1 \mu \mathrm{m}$. Refractive indices of olivine are used and we assumed $Q_{\mathrm{CO}}=$ $4 \times 10^{27}$ molecules s $^{-1}$.

dust size and size-dependent velocity distribution as used for the PACS data.

On 13 March 2013 at 06:00 UT, on the same date as PACS observations, an $A f \rho$ value of $\sim 900 \mathrm{~cm}$ was measured from $R$ band photometry by the consortium of amateur astronomers CARA (Milani \& Bryssinck, personal communication). This $A f \rho$ value was computed in an aperture of $5^{\prime \prime}$ radius $(15000 \mathrm{~km})$, approximately of the same size as the PACS pixels. For comparison, Bodewits et al. (2013) derived an $A f \rho$ normalized to a phase angle of $0^{\circ}$ of $1590 \mathrm{~cm}$ on 11 March 2013 from $V$-band magnitudes measured in an aperture of $10^{\prime \prime}$ radius. Considering the applied phase function of $\beta(\phi)=0.64 \beta(0)$ for $\phi=12.7^{\circ}$ (Schleicher 2010) and possible short-term variations, the $A f \rho$ value inferred by Bodewits et al. (2013) is consistent with the value measured by the CARA project.

In Table 3, the dust production rates derived from Af $\rho$ are computed for two assumptions on the minimum grain size $a_{\min }=$ 0.1 and $1 \mu \mathrm{m}$. Indeed, for steep size distributions, $a_{\mathrm{min}}$ affects the results, in contrast to the $Q_{\text {dust }}$ determination from PACS data which does not depend much on $a_{\text {min }}$. We assumed the geometric albedo of the grains to be 0.04 , consistent with calculations made by Hanner et al. (1981) for fluffy particles.

Comparison between the PACS and Af $\rho$-derived $Q_{\text {dust }}$ (Table 3) suggests a steep size distribution with $\alpha$ between -3.5 and -4 , depending on the material and minimum size considered. Figure 5 displays the comparison for olivine grains and the high activity case. We find that the best fit to both PACS 70/160 $\mu \mathrm{m}$ data and $A f \rho$ data is obtained for olivine grains, and a size index between -3.9 and -3.6 (Fig. 5). For these parameters, $Q_{\text {dust }}$ is $13 \mathrm{~kg} \mathrm{~s}^{-1}$ or $50-80 \mathrm{~kg} \mathrm{~s}^{-1}$, for the low and high activity cases, respectively. Similar conclusions are obtained using the refractive indices of carbon grains.

We recognise that the above results are assumptiondependent. For example, if we use a lower nucleus radius or a different grain density, it impacts the inferred dust production rate significantly. In the case of a nucleus radius assumed to be $R_{\mathrm{N}}=1 \mathrm{~km}$, the inferred dust production rate is 20-30 and $65-165 \mathrm{~kg} \mathrm{~s}^{-1}$ for the low and high activity case, respectively, where the low and high values in the ranges refer to calculations for carbon and olivine grains, respectively. As for the comparison between optical and PACS data, it rests upon the albedo of the grains, which might be, e.g., one order of magnitude lower than assumed if the grains are highly porous (Lacerda \& Jewitt 2012, and references therein).

\section{Conclusions}

We have presented a detailed analysis of our Herschel Space Observatory and IRAM observations of comet C/2012 S1 (ISON). The observations were performed in March and April 2013 when the comet was at a distance of approximately $4.5 \mathrm{AU}$ from the sun on the inbound portion of its orbit.

Our main findings are summarised below:

1. While $\mathrm{H}_{2} \mathrm{O}$ was not detected by the Herschel HIFI instrument, we derived a sensitive $3 \sigma$ upper limit of $Q_{\mathrm{H}_{2} \mathrm{O}}<$ $3.5 \times 10^{26}$ molecules s $^{-1}$.

2. We obtained a marginal $3.2 \sigma$ detection of $\mathrm{CO}$ with the IRAM $30 \mathrm{~m}$ telescope, corresponding to a $\mathrm{CO}$ production rate of $Q_{\mathrm{CO}}=3.5 \times 10^{27}$ molecules s $^{-1}$.

3. Our Herschel PACS measurements show a clear detection of the coma and tail in both the $70 \mu \mathrm{m}$ and $160 \mu \mathrm{m}$ maps. The length of the tail observed at $70 \mu \mathrm{m}$ images can be explained by the presence of grains with ages older than 60 days. The radial profile in the inner coma observed at $160 \mu \mathrm{m}$ suggests an excess of slow particles. With an expected terminal velocity of $100-\mu \mathrm{m}$ dust particles being typically $10 \mathrm{~m} \mathrm{~s}^{-1}$ (estimated from our models) the travel time of such slow particles through the PSF is $\sim 15$ days. 
4. Under the assumption of a $2-\mathrm{km}$ radius nucleus, we infer dust production rates in the range $10-13 \mathrm{~kg} \mathrm{~s}^{-1}$ or $40-70 \mathrm{~kg} \mathrm{~s}^{-1}$, depending whether a low $\left(4 \times 10^{26}\right.$ molecules $\left.^{-1}\right)$ or high $\left(4 \times 10^{27}\right.$ molecules $\left.^{-1}\right)$ gaseous activity from the nucleus surface is considered. Size indices between -4 and -3.6 are suggested.

5. A comparison with optical data taken on the same date as our Herschel PACS measurements was performed. We obtained a satisfactory fit to both PACS $70 / 160 \mu \mathrm{m}$ data and CARA Af $\rho$ data in the case of olivine grains whereby we find that a size index between -3.9 and -3.6 best matches the data. For these parameters, $Q_{\text {dust }}$ is $13 \mathrm{~kg} \mathrm{~s}^{-1}$ or $50-80 \mathrm{~kg} \mathrm{~s}^{-1}$, for the low and high activity cases, respectively.

6. In the scenario of weak surface activity $\left(Q_{\mathrm{CO}}=\right.$ $2.5 \times 10^{26}$ molecules s $^{-1}$, as derived from the magnitude $/ Q_{\mathrm{CO}}$ correlation established from Hale-Bopp data), we derive a dust-to-gas ratio of $\sim 1$ for $R_{\mathrm{N}}=2 \mathrm{~km}$, and in the range 2-3 for $R_{\mathrm{N}}=1 \mathrm{~km}$. Dust-to-gas ratios lower than 1 are inferred in the high activity case.

Acknowledgements. We wish to thank the Herschel Project Scientist and Time Allocation Committee for awarding $5 \mathrm{~h}$ of Director Discretionary Time for this observation. We thank Andy Pollock for his final proof-reading checks. We thank G. Milani and E. Bryssinck for providing us Af $\rho$ measurements on behalf the Cometary ARchive for Afrho (CARA) consortium (http://cara.uai.it/), and R. Ligustri who performed the observation that we used. C.S. has received funding from the European Union Seventh Framework Programme (FP7/20072013) under grant agreement No. 268421.

\section{References}

A'Hearn, M. F., Schleicher, D. G., Millis, R. L., Feldman, P. D., \& Thompson, D. T. 1984, AJ, 89,579

Biver, N. 2001, International Comet Quarterly, 23, 85

Biver, N., Bockelée-Morvan, D., Colom, P., et al. 2002, Earth Moon and Planets, 90, 5

Biver, N., Bockelée-Morvan, D., Crovisier, J., et al. 2007, Planet. Space Sci., 55, 1058

Bockelée-Morvan, D., Hartogh, P., Crovisier, J., et al. 2010, A\&A, 518, L149

Bodewits, D., Farnham, T., \& A'Hearn, M. F. 2013, Central Bureau Electronic Telegrams, 3608, 1

Burns, J. A., Lamy, P. L., \& Soter, S. 1979, Icarus, 40, 1

Crifo, J. F., \& Rodionov, A. V. 1997, Icarus, 127, 319
Crovisier, J., Leech, K., Bockelée-Morvan, D., et al. 1997, Science, 275, 1904 Davidsson, B. J. R., Gutiérrez, P. J., \& Rickman, H. 2007, Icarus, 187, 306

de Graauw, T., Helmich, F. P., Phillips, T. G., et al. 2010, A\&A, 518, L6

Dorschner, J., Begemann, B., Henning, T., Jaeger, C., \& Mutschke, H. 1995 , A\&A, 300, 503

Edoh, J. H. 1983, Ph.D. Thesis, Univ. Arizona

Finson, M. L., \& Probstein, E. F. 1968, ApJ, 154, 353

Fornasier, S., Lellouch, E., Müller, T., et al. 2013, A\&A, 555, A15

Gunnarsson, M., Bockelée-Morvan, D., Biver, N., Crovisier, J., \& Rickman, H. 2008, A\&A, 484, 537

Hartogh, P., Crovisier, J., de Val-Borro, M., et al. 2010, A\&A, 518, L150

Hanner, M. S., Giese, R. H., Weiss, K., \& Zerrull, R. 1981, A\&A, 104, 42

Jorda, L. 1995, Ph.D. Thesis, University of Paris 7

Jorda, L., Lamy, P., Faury, G., et al. 2007, Icarus, 187, 208

Jewitt, D., \& Luu, J. 1990, ApJ, 365, 738

Kiss, C., Müller, T. G., Vilenius, E., et al. 2013, Exp. Astron., accepted [arXiv: 1309.4212]

Lacerda, P., \& Jewitt, D. 2012, ApJ, 760, L2

Li, J.-Y., Weaver, H. A., Kelley, M. S., et al. 2013, Central Bureau Electronic Telegrams, 3496, 1

Lutz, D. 2012, PACS ICC technical note, PICC-ME-TN-033, http://herschel.esac.esa.int/twiki/bin/view/Public/ PacsCalibrationWeb?template=viewprint

Lisse, C. M., Vervack, R. J., Weaver, H. A., et al. 2013, Central Bureau Electronic Telegrams, 3591, 2

Meech, K. J., Yang, B., Ansdell, M., et al. 2013, ApJ, submitted

Müller, T. G., Okumura, K., \& Klaas, U. 1. 2011, PACS technical report PICC-ME-TN-038, v1.0: http://herschel.esac.esa.int/ -twiki/-pub/-Public/-PacsCalibrationWeb/-cc/report/v1.pdf

Müller, T. G., O’Rourke, L., Barucci, A. M., et al. 2012, A\&A, 548, A36

Nevski, V., \& Novichonok, A. 2012, IAU Circ., 3238

Niimi, R., Kadono, T., Tsuchiyama, A., et al. 2012, ApJ, 744, 18

Ootsubo, T., Kawakita, H., Hamada, S., et al. 2012, ApJ, 752, 15

O'Rourke, L., Müller, T., Valtchanonv, I.,et al. 2012, Planet. Space Sci., 66, 192

O'Rourke, L., Snodgrass, C., de Val Borro, M., et al. 2013, ApJ, 774, 13

Ott, S. 2010, ASP Conf. Ser., 434, 139

Pilbratt, G. L., Riedinger, J. R., Passvogel, T., et al. 2010, A\&A, 518, L1

Poglitsch, A., Waelkens, C., Geis, N., et al. 2010, A\&A, 518, L2

Richardson, J. E., Melosh, H. J., Lisse, C. M., \& Carcich, B. 2007, Icarus, 191, 176

Schleicher, D. 2010, http://asteroid.lowell.edu/comet/dustphase. html

van de Hulst, H. C. 1981, Light scattering by small particles (New York: Dover)

Thomas, P. C., A'Hearn, M. F., Veverka, J., et al. 2013, Icarus, 222, 550

Weiler, M., Rauer, H., Knollenberg, J., Jorda, L., \& Helbert, J. 2003, A\&A, 403, 313

Zakharov, V., Bockelée-Morvan, D., Biver, N., Crovisier, J., \& Lecacheux, A. 2007, A\&A, 473, 303 